\title{
Study on chemical, UV and gamma radiation-induced grafting of 2-hydroxyethyl methacrylate onto chitosan
}

\author{
M.H. Casimiro ${ }^{\mathrm{a}, *}$, M.L. Botelho ${ }^{\mathrm{a}}$, J.P. Leal ${ }^{\mathrm{b}, \mathrm{c}}$, M.H. Gil $^{\mathrm{d}}$ \\ ${ }^{a}$ Department of Physics, Nuclear and Technological Institute, E.N. 10, 2686-953 Sacavém, Portugal \\ ${ }^{\mathrm{b}}$ Department of Chemistry, Nuclear and Technological Institute, E.N. 10, 2686-953 Sacavém, Portugal \\ ${ }^{\mathrm{c}}$ Department of Chemistry and Biochemistry, Faculty of Sciences, University of Lisboa, 1749-016 Lisboa, Portugal \\ ${ }^{\mathrm{d}}$ Department of Chemical Engineering, Faculty of Science and Technology, University of Coimbra, Pólo II Pinhal de Marrocos, \\ 3030-290 Coimbra, Portugal
}

Received 18 February 2004; accepted 13 April 2004

\begin{abstract}
In the present study, 2-hydroxyethyl methacrylate has been grafted onto chitosan by using either chemical initiation, or photo-induction or gamma radiation-induced polymerisation, all under heterogeneous conditions. The evidence of grafting was provided by Fourier transform infrared spectroscopy and thermal analysis.

The results concerning the effect of initiator concentration, initial monomer concentration and dose rate influencing on the yield of grafting reactions are presented. These suggest that gamma irradiation is the method that leads to higher yields of grafting.
\end{abstract}

(C) 2004 Elsevier Ltd. All rights reserved.

Keywords: Chitosan; HEMA; Graft copolymerisation

\section{Introduction}

Chitosan $(\beta-1,4$-glucosamine $)$ is a polysaccharide of natural origin, obtained by full or partial deacetylation of chitin, a very abundant natural polymer that can be derived from the outer shells of crustaceans. Due to its numerous and interesting biological properties such as biocompatibility, biodegradability and non-toxic properties, chitosan and its derivatives have become useful polysaccharides in biomedical and environmental areas (Luyen and Huong, 1996; Burke et al., 2002). However, in order to make the polymer adequate to each application, modifications on the chitosan structure are needed.

Of the possible physical-chemical modifications of chitosan, graft copolymerisation can provide materials

\footnotetext{
*Corresponding author. Fax: +351-219941525.

E-mail address: casimiro@itn.mces.pt (M.H. Casimiro).
}

with the desired properties through the appropriate selection of the molecular characteristics of side chain to be grafted. In this way graft copolymerisation is commonly used as a general method for polymer modification (Alves da Silva et al., 1987; Kurika, 1996; Pengfei et al., 2001; Khor and Lim, 2003).

Some of previous studies showed that the presence of 2-hydroxyethyl methacrylate (HEMA) in copolymers improve the biocompatibility of the materials (Carenza, 1992; Montheard et al., 1992; Ferreira et al., 1998).

The use of chitosan-based copolymers and the use of HEMA as a grafting monomer onto a range of polymeric substrates have been reported with increasing success as biomaterials (Shantha et al., 1995; Ahn et al., 2002; Zhang et al., 2002). Nevertheless the comparison between the usual radical polymerisation methods to obtain the mentioned copolymer has not yet been described.

Our attention has been focused on efficient and wellcontrolled grafted copolymerisation methods onto 
chitosan in order to obtain a biocompatible copolymer for controlled release of drugs. Here we report the comparative results of the graft copolymerisation behaviour of HEMA onto chitosan induced by chemical initiator $\left(\mathrm{Ce}^{4+}\right)$, by photo-induction with UV light and by gamma radiation under heterogeneous conditions. Evidence of grafting has been provided by Fourier transform infrared spectroscopy (FTIR) spectroscopy and thermal analysis.

\section{Experimental}

\subsection{Materials}

Chitosan medium molecular weight $\left(1.9 \times 10^{5}-\right.$ $\left.3.1 \times 10^{5} \mathrm{Da}\right)$ was obtained from Aldrich Chemical Company, Inc., Milwaukee, USA. This was triturated $(500<\phi<800 \mu \mathrm{m})$ and dried under vacuum at $313 \mathrm{~K}$. Hydroxyethyl methacrylate (HEMA), stabilized, 98\%, was obtained from ACROS Organics, Belgium, and used as received.

The chemical initiator, cerium ammonium nitrate, was obtained from $\mathrm{BDH}, \mathrm{UK}$, and the photo-activator, isopropyl thioxanthone (ITX), from Aldrich Chemical Company, Inc., Milwaukee, USA. Acetone and methanol analytical grade obtained from Riedel-deHaen, Germany, were used as received. Nitric acid was purchased from Merck, Germany.

\subsection{Procedures}

\subsubsection{Chemical grafting reaction}

The $0.5 \mathrm{~g}$ of chitosan was suspended in $30 \mathrm{~cm}^{3}$ of an acetone solution containing HEMA $0.82 \mathrm{M}(10 \% \mathrm{~V} / \mathrm{V})$, nitric acid $1.6 \times 10^{-1} \mathrm{M}$ and cerium ammonium nitrate $9.10 \times 10^{-4}, 4.56 \times 10^{-3}$ and $1.82 \times 10^{-2} \mathrm{M}(0.05,0.25$ and $1 \% \mathrm{w} / \mathrm{V}$, weight per volume), were treated at $323 \mathrm{~K}$, in the presence of air, for different intervals of time.

\subsubsection{UV grafting reaction}

The $0.5 \mathrm{~g}$ of chitosan was suspended in $100 \mathrm{~cm}^{3}$ of an acetone/water (1:1) solution containing the photoactivator $10^{-3} \mathrm{M}$ and HEMA $0.82 \mathrm{M}$. The dispersions were irradiated in the presence of air with a water-cooled medium pressure UV lamp assembly, which provided an output of $80 \mathrm{~W} / \mathrm{cm}$ for different intervals of time, at room temperature.

\subsubsection{Gamma irradiation grafting}

The $0.6 \mathrm{~g}$ of chitosan was suspended in $20 \mathrm{~cm}^{3}$ of a HEMA solution $0.41,0.82$ and $1.23 \mathrm{M}(5 \%, 10 \%$ and $15 \% \mathrm{~V} / \mathrm{V})$ in methanol. The dispersions were purged with nitrogen and then $\gamma$-irradiated at 3.8 or $7.4 \mathrm{kGy} \mathrm{h}^{-1}$ using a ${ }^{60} \mathrm{Co}$ gamma source, for several different intervals of time, at room temperature. Samples were irradiated in a position parallel to the irradiator and amber and red dosimeters (Harwell) were used to monitor absorbed dose. After reaction, all bulk solutions and grafted chitosan powder were transferred into an excess of methanol. The homopolymer was removed by extraction in a soxhlet for $6 \mathrm{~h}$ and subsequently dried to constant weight in vacuum at $313 \mathrm{~K}$. Each system was studied in triplicate.

\subsection{Grafting parameters}

For chitosan copolymers, the grafting parameters were obtained by using the following expressions:

$$
\begin{aligned}
& \text { Yield of grafting }(\%) \\
& =\frac{\text { wt. of graft coploymer }- \text { wt. of chitosan }}{\text { wt. of chitosan }} \times 100,
\end{aligned}
$$

Efficiency of grafting (\%)

$$
=\frac{\text { wt. of graft copolymer }- \text { wt. of chitosan }}{\text { wt. of initial monomer }} \times 100 \text {. }
$$

\subsection{Thermal analysis}

The thermal properties of the polymer systems were evaluated by TGA. These assays were carried out on a TA Instruments 951 Thermogravimetric Analyser in nitrogen atmosphere at a heating rate of $10^{\circ} \mathrm{C} / \mathrm{min}$ over the temperature range of $30-500^{\circ} \mathrm{C}$.

\subsection{Infrared spectroscopy}

IR absorption spectra of HEMA, poly (HEMA), chitosan and the obtained copolymeric samples were studied by FTIR. IR spectra were recorded in $\mathrm{KBr}$ disks on a Perkin-Elmer 1600 Series FTIR spectrometer. All spectra were recorded at ambient temperature at the resolution of $16 \mathrm{~cm}^{-1}$ and 4 times scanning.

\section{Results and discussion}

\subsection{Effect of the copolymerisation methods on the yield of grafting}

The results in Fig. 1 show that the yield of grafting of HEMA onto chitosan is higher when the $\gamma$ irradiation method is used. It is also possible to observe that there is a "plateau" after $1 \mathrm{~h}$ reaction in the case of chemical initiation, $3 \mathrm{~h}$ when $\gamma$ irradiation is used and $4 \mathrm{~h}$ when the grafting reaction is carried out with UV.

The radical polymerisation methods involve the formation of active sites at the backbone polymer, which become the points of initiation for the monomer side chains (Dessouki et al., 1999). When $\gamma$ radiation is 


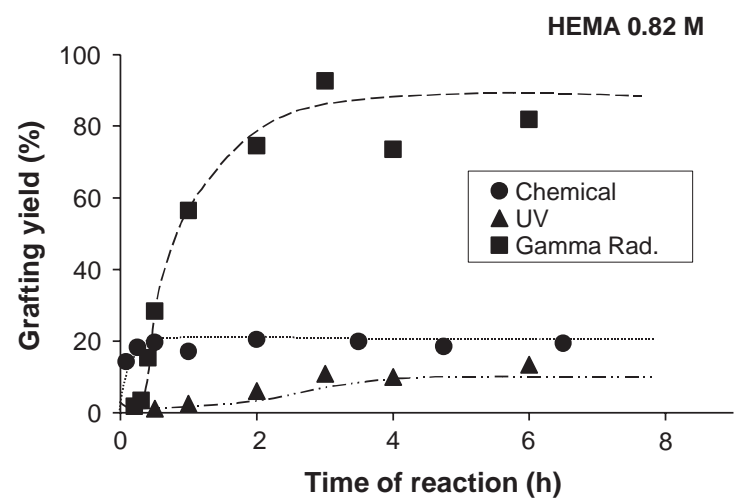

Fig. 1. HEMA graft copolymerisation onto chitosan induced by chemical, UV and gamma radiation (dose rate $7.4 \mathrm{kGy} \mathrm{h}^{-1}$ ).

used to induce the graft polymerisation the energy deposited takes place non-selectively and uniformly in the reaction system. Thus this promotes an uniform activation of the entire system and consequently higher grafting yields are achieved.

In the next sections we will consider the detailed results obtained with the various routes of initiation.

\subsection{Chemical grafting initiation - effect of the initiator concentration}

When chemical initiation is used, the effect of the initiator concentration was evaluated for concentrations of $0.05 \%, 0.25 \%$ and $1 \% \mathrm{w} / \mathrm{V}$ with a monomer initial concentration of $0.82 \mathrm{M}$. Results showed a similar behavior to the one presented in Fig. 1 with $1 \% \mathrm{w} / \mathrm{V}$ of initiator presenting the maximum yield of grafting over the range of $15-20 \%$. Thus in the studied range there is no clear relation between the grafting yield and the initiator concentration.

Ceric ion $\left(\mathrm{Ce}^{4+}\right)$ is a strong redox initiator that can oxidize the polysaccharide with the production of a free radical on the ring (Don et al., 2002). Thus, a higher concentration of $\mathrm{Ce}^{4+}$ is expected to activate more sites at the system in study. However, a higher quantity of $\mathrm{Ce}^{4+}$ can also promote increasing in homopolymer reaction. In this way, to better understand the behavior of the grafting of HEMA onto chitosan using chemical initiation and determine the optimum concentration of $\mathrm{Ce}^{4+}$, further studies would be needed. Moreover, as this initiation method did not present the higher grafting yields, particularly attention was then given to the gamma radiation-induced polymerisation.

\section{3. $\gamma$-Irradiation-effect of monomer concentration}

The relation between grafting yield and the initial monomer concentration in bulk solution is shown in Fig. 2. Three concentrations of monomer were used at a dose rate of $7.4 \mathrm{kGy} \mathrm{h}^{-1}$. It can be seen that within the same dose rate, the increase of monomer concentration is accompanied by a significant increase in the degree of grafting. According to Dessouki et al. (1999) this behavior can be attributed to diffusion mechanisms as well as a bigger amount of HEMA molecules initially available for reaction. However this might enhance not only the copolymerisation reaction but also the homopolymerisation reaction. This is pointed out by the percent grafting efficiency that present the values $38 \%$, $23 \%$ and $21 \%$ for initial HEMA concentration of $5 \%$, $10 \%$ and $15 \% \mathrm{~V} / \mathrm{V}$, respectively. The grafting efficiency values were obtained considering the steady-state medium value for each HEMA concentration.

Although there are not enough experimental values on the first hour of irradiation that can lead to a clear kinetics, the variation of the average rate of grating of HEMA onto chitosan by $\gamma$-radiation-induced polymerisation $\left([\mathrm{HEMA}]_{i}=0.82 \mathrm{M}, \mathrm{DR}=7.4 \mathrm{kGy} \mathrm{h}^{-1}\right.$ ) is presented in Fig. 3. It can be noted that after an initial induction period (with low rates) a sudden increase happens followed by a fast decrease that reaches very low values after $1-2 \mathrm{~h}$. If the initial points (induction period) were excluded (some time is needed also for the monomer inhibitor consumption), a power function fits quite well to the experimental points $\left(r^{2}=0.94\right.$; average rate $=0.0769 \times$ time $\left.^{-1.289}\right)$. This order of magnitude is consistent with the time needed to achieve the plateau (see Fig. 2). Also in Fig. 2, for the case of higher concentration of HEMA (higher viscosity) and for doses above $40 \mathrm{kGy}$, what could be the beginning of a Tromsdorf-Norrish effect can be noticed. However, to be sure about it, higher doses on the same conditions would be needed.

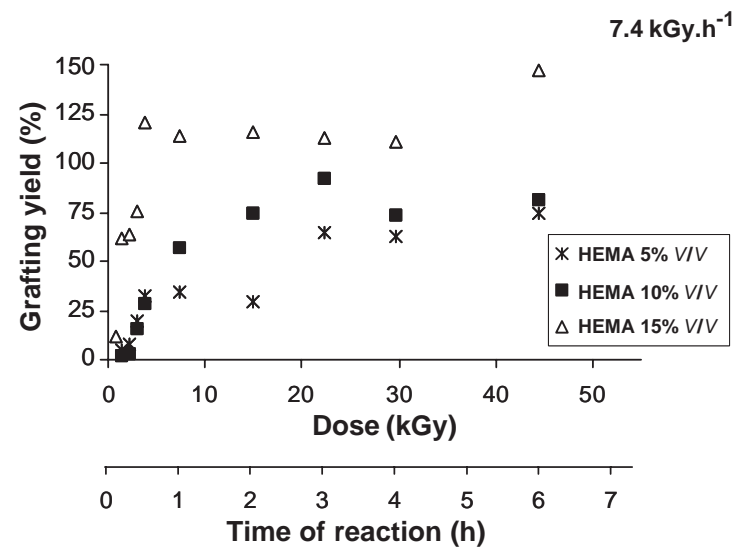

Fig. 2. Effect of HEMA concentration on the grafting of HEMA onto chitosan (polymerisation induced by $\gamma$-radiation). The two scales (dose and time) are shown to make clear the equivalence between them when the dose rate is kept constant (in this case $7.4 \mathrm{kGy} \mathrm{h}^{-1}$ ). $5 \%, 10 \%$ and $15 \% \mathrm{~V} / \mathrm{V}$ corresponds to $0.41,0.82$ and $1.23 \mathrm{M}$, respectively. 


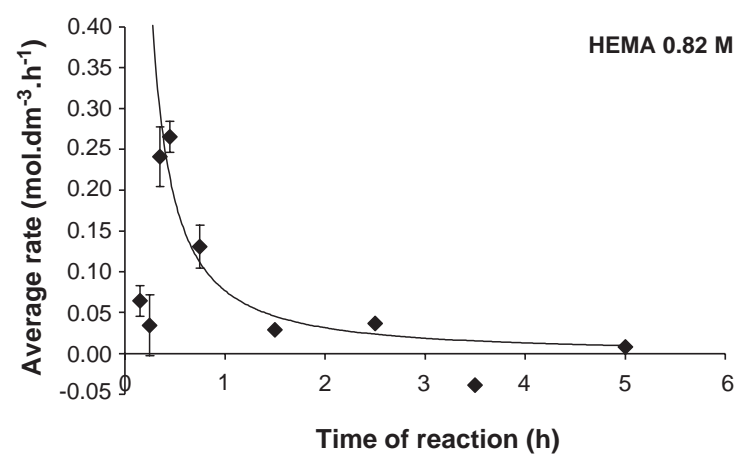

Fig. 3. Variation of the average rate of grating of HEMA onto chitosan by $\gamma$-radiation-induced polymerisation ([HE$\mathrm{MA}]_{i}=0.82 \mathrm{M}, \mathrm{DR}=7.4 \mathrm{kGy} \mathrm{h}^{-1}$ ).

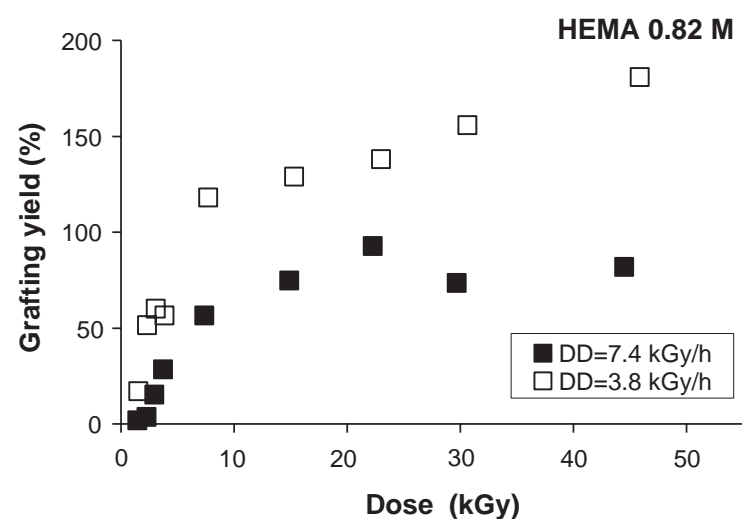

Fig. 4. Effect of dose rate on the grafting of HEMA onto chitosan.

\section{4. $\gamma$-Irradiation - effect of the dose rate}

Fig. 4 shows the effect of dose rate on the grafting of HEMA onto chitosan. The results indicate that low dose rate $\left(3.8 \mathrm{kGy} \mathrm{h}^{-1}\right)$ leads to higher grafting yields than the same radiation doses of higher rate $\left(7.4 \mathrm{kGy} \mathrm{h}^{-1}\right)$. This is connected not only with the high radical density due to the higher dose rate, which favors second-order radicalradical reaction (Kiatkamjornwong et al., 2000), but also due to the additional degradation of chitosan as a consequence of the predominance of main chain scission over crosslinking (Ulanski and Rosiak, 1992). The experimental results obtained with the polymeric matrix (chitosan) without HEMA monomer seem to be in agreement with last authors results since experiments have led to a continuous reduction of chitosan mass with the irradiation time (or dose). This mass reduction was more significant for the higher dose rate: for $4 \mathrm{~h}$ of irradiation the mass loss was approximately of $3 \%$ for the $3.8 \mathrm{kGy} \mathrm{h}^{-1}$ and $5 \%$ for the $7.4 \mathrm{kGy} \mathrm{h}^{-1}$ dose rate.

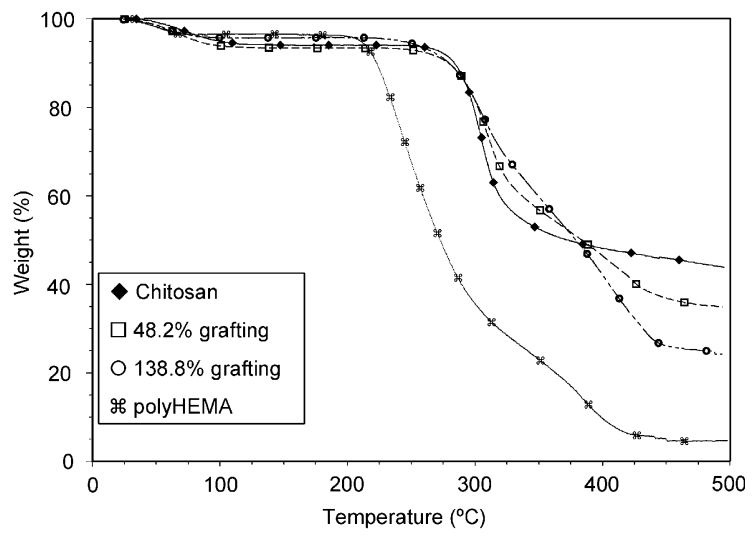

Fig. 5. TGA thermograms of chitosan, poly(HEMA) and copolymers with different grafting yields $\left([\text { HEMA }]_{i}=0.82 \mathrm{M}\right.$, $\mathrm{DR}=3.8 \mathrm{kGy} \mathrm{h}^{-1}$ ).

\subsection{TGA analysis}

To evaluate the thermal properties of the polymers, the samples were characterised by TGA. Fig. 5 shows the weight loss curves of chitosan, poly(HEMA) and two copolymers obtained by gamma irradiation with different grafting yields.

It was observed that the initial thermal decomposition of chitosan took place at $255^{\circ} \mathrm{C}$ whereas poly(HEMA) exhibited it at $195^{\circ} \mathrm{C}$. It was also observed that poly(HEMA) shows an almost complete weight loss at $500^{\circ} \mathrm{C}$, while chitosan only loose about $50 \%$ of the initial weight. The chitosan degradation mechanism is complex (includes the dehydration, deacetylation and chain scission) and is not well understood. However, the weight loss observed correspond to the losses observed by other authors (Kim et al., 2000; Don et al., 2002). For both copolymers its initial thermal decomposition started very near to that observed for chitosan. These results show that after the copolymerisation process the copolymers maintain the thermal stability characteristic of backbone structure (chitosan) even with high grafting yields. However, the copolymer curves show an increasing similarity with the poly(HEMA) curve with the increase in grafting yield, which suggests that grafted branches chain grow with the increase of grafting yield.

\subsection{FTIR analysis}

The FTIR analysis was based on the identification of absorption bands concerned with the vibrations of functional groups present in the molecules. The FTIR spectra of chitosan, poly(HEMA) and two copolymers with different grafting yields are shown in Fig. 6.

The continuously increasing peak near $750 \mathrm{~cm}^{-1}$, characteristic of the methacrylic polymers that exhibits here a strong $\mathrm{CH}_{2}$ rocking peak, can be observed in 


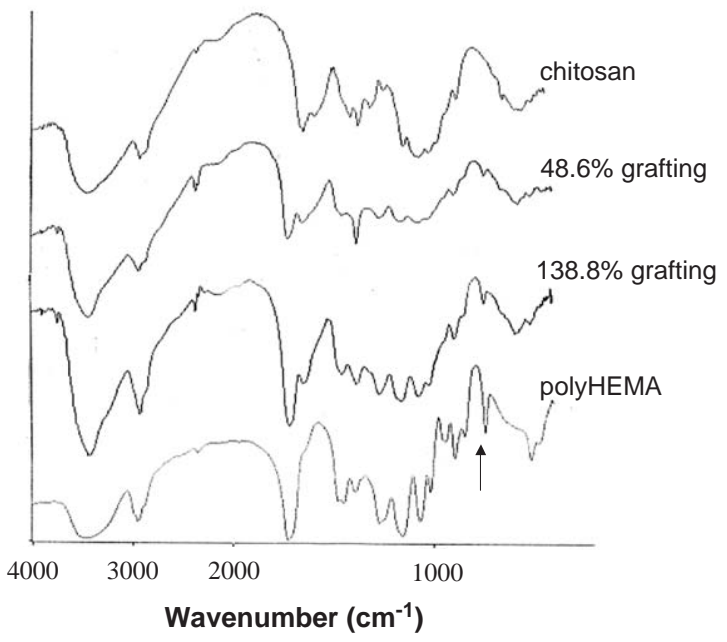

Fig. 6. FTIR spectra of chitosan, polyHEMA and copolymers with different grafting yields $\left([\mathrm{HEMA}]_{i}=0.82 \mathrm{M}\right.$, $\mathrm{DR}=3.8 \mathrm{kGy} \mathrm{h}^{-1}$ ); the arrow indicates the $\mathrm{CH}_{2}$ rocking peak characteristic of methacrylic polymers.

spectra of copolymers with increasing grafting. These results seem to confirm that poly(HEMA) grafted branches grow with the grafting procedure.

\section{Conclusions}

The obtained results suggest that graft copolymerisation induced by gamma radiation is the method that leads to higher grafting yields. Since the efficiency of grafting is depending on initial monomer concentration and on dose rate, the grafting yield can be controlled by appropriate selection of grafting conditions.

\section{Acknowledgements}

This work has been supported by Operational Programme of Science, Technology and Innovation from Third European Community Framework Programme and from Foundation of Science and Technology, Portugal (Grant SFRH/BD/2862/2000).

\section{References}

Ahn, J.-S., Choi, H.-K., Chun, M.-K., Ryu, J.-M., Jung, J.-H., Kim, Y.-U., Cho, C.-S., 2002. Release of triamcinolone acetonide from mucoadhesive polymer composed by chitosan and poly(acrylic acid) in vitro. Biomaterials 23, 1411-1416.
Alves da Silva, M., Gil, M.H., Lapa, E., Guthrie, J.T., 1987. Photosensitized grafting of acrylamide and hydroxyethtyl methacrylate onto cellulose. J. Appl. Polym. Sci. 34, 871-880.

Burke, A., Yilmaz, E., Hasirci, N., Yilmaz, O., 2002. Iron removal from solution through adsorption on chitosan. J. Appl. Polym. Sci. 84, 1185-1192.

Carenza, M., 1992. Recent achievements in the use of radiation polymerisation and grafting for biomedical applications. Radiat. Phys. Chem. 39 (6), 485-493.

Dessouki, A.M., El-Tahawy, M., El-Boohy, H., El-Mongy, S.A., Badawy, S.M., 1999. Chemical reactive paper prepared by radiation-induced graft copolymerisation-I. Radiat. Phys. Chem. 54, 627-635.

Don, T.-M., Chuang, C.-Y., Chiu, W.-Y., 2002. Studies on the degradation behavior of chitosan-g-poly(acrylic acid) copolymers. Proceedings of the 9th Asian-Pacific Confederation of Chemical Engineering Congress, New Zealand, September/October 2002.

Ferreira, L.M., Rocha, J.M.S., Andrade, M.E., Gil, M.H., 1998. Preparation and characterisation of polyethylene based graft copolymers. Applications in the immobilization of enzymes. Radiat. Phys. Chem. 52 (1-6), 207-212.

Khor, E., Lim, L.Y., 2003. Implantable applications of chitin and chitosan. Biomaterials 24, 2339-2349.

Kiatkamjornwong, S., Chomsaksakul, W., Sonsuk, M., 2000. Radiation modification of water absorption of cassava starch by acrylic acid/acrylamide. Radiat. Phys. Chem. 59, 413-427.

Kim, S.Y., Cho, S.M., Lee, Y.M., Kim, S.J., 2000. Thermoand $\mathrm{pH}$-responsive behaviors of graft copolymer and blend based on chitosan and $N$-isopropylacrylamide. J. Appl. Polym. Sci. 78, 1381-1391.

Kurika, K., 1996. Chitin and chitosan graft copolymers. In: J.C. Salamone (Ed.), Polymeric Materials Encyclopedia, Vol. 2. CRC Press, New York, pp. 1205-1208.

Luyen, D.V., Huong, D.M., 1996. Chitin and derivatives. In: J.C. Salamone (Ed.), Polymeric Materials Encyclopedia, Vol. 2. CRC Press, New York, pp. 1208-1217.

Montheard, J.-P., Chatzopoulos, M., Chappard, D., 1992. 2hydroxyethyl methacrylate (HEMA): chemical properties and applications in biomedical fields. J.M.S-Rev. Macromol. Chem. Phys. 32 (1), 1-34

Pengfei, L., Maolin, Z., Jilan, W., 2001. Study on radiationinduced grafting of styrene onto chitin and chitosan. Radiat. Phys. Chem. 61 (2), 149-153.

Shantha, K.L., Udaya Bala, Panduranga Rao, K., 1995. Tailormade chitosans for drug delivery. Eur. Polym. J. 31 (4), 377-382.

Ulanski, P., Rosiak, J., 1992. Preliminary studies on radiationinduced changes in chitosan. Radiat. Phys. Chem. 39 (1), 53-57.

Zhang, M., Li, X.H., Gong, Y.D., Zhao, N.M., Zhang, X.F., 2002. Properties and biocompatibility of chitosan films modified by blending with PEG. Biomaterials 23, 2641-2648. 